\title{
Physical Activity and Exercise
}

\author{
Michael Riddell ${ }^{1}$, Sophie Pollack ${ }^{2}$, Homadis Shojaei ${ }^{1}$, Joshua Kalish ${ }^{2}$, and Howard Zisser $^{2}$
}

\section{Introduction}

$\mathbf{E}^{\mathrm{x}}$ XERCISE HAS BEEN PRESCRIBED FOR DIABETES treatment since at least 600 B.C. The early East Indian text, the Shushruta, described a reduction in the sweetness of urine from diabetic patients after exercise. One might think that very little could be left to discover in the field of exercise and diabetes, yet surprisingly this is far from the truth. Ongoing research is refining the exercise prescription for patients of all ages, with the main types of diabetes (gestational, type 1, and type 2) and discovering new ways in which exercise has benefits. Alterations in metabolism caused by diabetes and new types of exercise modalities are also actively being researched. A search of several hundred articles on exercise published between July 1, 2012, to June 30, 2013, uncovered the following 9 articles we felt had the most relevance to patients with diabetes or prediabetes.

\section{Work volume and strength training responses to resistive exercise improve with periodic heat extraction from the palm}

Grahn DA, Cao VH, Nguyen CM, Liu MT, Heller HG

Department of Biology, Stanford University, Stanford, CA

J Strength Cond Res 2012; 26: 2558-69

\section{Background and Rationale}

It has previously been shown that attenuating the rise in the body's core temperature improves aerobic exercise endurance capacity in athletes. Temperatures above the optimal range appear to reduce contractile force production capacity of skeletal muscles. Thus, heat extraction may be one way to help increase exercise performance and involvement. In this study, the benefits of core-temperature reduction are investigated with respect to resistance exercise capacity in healthy men.

\section{Methods}

Sixty-seven healthy men were self-selected within the age range of 19-23 years. Three protocols were carried out in a crossover design. The protocols lasted from 6 to 12 weeks. Protocol 1: Core temperature was increased by treadmill exercise and decreased by palm cooling in order to study effects of changing temperature on bench press volume. Protocol 2: Palm cooling was used as a means of extracting heat to study its influence on pull-up and bench-press work volume training response. Protocol 3: Strength-training response to changes in body core temperature was assessed with and without palm cooling. In all protocols, heat extraction was performed by a palm-cooling device, which comprised a stainless steel plate with a temperature-controlled water bath located underneath the plate. Since heat dissipation for the whole body is done partially through the palms, cooling the palms results in significant core-temperature reduction that can be monitored via a thermocouple probe.

\section{Results}

Protocol 1: Palm cooling reduced the rise in core temperature that occurred with treadmill exercise (esophageal temperature after treadmill exercise $=38.4 \pm 0.2^{\circ} \mathrm{C}$ with palm cooling vs. $39.0 \pm 0.1^{\circ} \mathrm{C}$ without palm cooling, $n=8 ; p=0.01$ ). Bench press volume was higher with interset palm cooling during rest compared with interset rest only (total reps/trial: $42 \pm 7$ reps after palm cooling vs. $36 \pm 7$ reps after no cooling; $p<0.001$ ). Protocol 2: Subjects with and without prior pull-up training showed greater rate of training response with palm cooling than without palm cooling. The improvement rate was $13 \pm 8$ pullups/trial with palm cooling compared with $3 \pm 4$ pull-ups/trial without palm cooling. The same trend was observed for bench press exercise. The final-to-initial total repetition ratio with interset palm cooling was $1.4 \pm 0.1$ and without interset palm cooling was $1.1 \pm 0.1$. Protocol 3: Interset palm cooling increased the strength training response (an increase by $\sim 13 \mathrm{~kg}$ in the fourth set), whereas there were no observed changes in strength with training using interset rest only.

\section{Conclusion}

Palm cooling, as a means of improved core-temperature regulation, improves work volume and resistance exercise

\footnotetext{
${ }^{1}$ School of Kinesiology and Health Science, York University, Toronto, Canada.

${ }^{2}$ Sansum Diabetes Research Institute, Santa Barbara, CA.
} 
training response. Temperature regulation may potentially be an effective means of enhancing exercise and sport performance. The palm cooling device may be a breakthrough for training efficiency for athletes and to help them conquer their conditioning plateaus.

\section{Comment}

The study illustrates the effectiveness of the innovative palm-cooling technology for improving athletic performance by enhancing heat dissipation. This technology may have significant implications in terms of alleviation of symptoms of premature fatigue in patients with diabetes. Indeed, both impaired heat dissipation (1) and premature fatigue (2) are characteristic of people with type 2 diabetes mellitus (T2DM). In addition, the cooccurring complications as well as the medications required for their treatment appear to reduce skin blood flow and lower sweating volume, which impairs cooling. Therefore, the likelihood of heat-related diseases is higher in diabetic patients than in the healthy population (1). One key drawback of the study design, however, is that for the first part of the training experiment, subjects had interset rest only, and for the following section subjects exercised with interset palm cooling at rest. The order effect would be biased toward favoring palm cooling's effect on training.

\section{Exercise dose and diabetes risk in overweight and obese children: a randomized control trial}

Davis $C L^{1}$, Pollock $N K^{1}$, Bassali $R^{1}$, Boyle $C A^{1}$, Waller $\mathrm{JL}^{2}$, Allison $\mathrm{JD}^{3}$, Dennis $B A^{4}$, Meléndez $A^{5}$, Gower $B A^{6}$

Departments of ${ }^{1}$ Pediatrics, ${ }^{2}$ Biostatistics, ${ }^{3}$ Radiology, and ${ }^{4}$ Medicine, Georgia Prevention Center, Institute for Public and Preventive Health, Medical College of Georgia, Augusta, GA; ${ }^{5}$ Polytechnic University of Madrid, Madrid, Spain; and ${ }^{6}$ Department of Nutrition Sciences, University of Alabama, Birmingham, AL

JAMA 2012; 308: 1103-12

\section{Background and Rationale}

Obesity and excess body weight are highly prevalent among U.S. children. It has been reasonably well established that regular exercise reduces the risk of both excess body adiposity and T2DM development in adults with prediabetes, but less is known about adolescents at risk for T2DM. The purpose of this study was to explore the relationship between various aerobic exercise volumes and T2DM risk factors in overweight and obese children. This information would allow health practitioners to more accurately prescribe exercise that is optimal for limiting T2DM risk factors in youth.

\section{Methods}

In this pretest-posttest randomized control trial, 222 sedentary obese or overweight children (7 to 11 years old) were recruited from 15 elementary schools. The duration of the study was 13 weeks for each cohort. The subjects were randomly assigned to control, low-dose aerobic exercise (20 min/ day), or high-dose aerobic exercise (40 min/day) and stratified by race and sex. An intention-to-treat analysis was used.
Average heart rates above $150 / \mathrm{min}$ were encouraged by giving "points," which were exchanged for prizes at the end of each week. The hormones testosterone and estradiol were measured in males and females, respectively, to control for the effects of puberty on insulin resistance. To compare intergroup outcomes, insulin area-under-the-curve (AUC) analysis, percent body fat, visceral fat mass, and aerobic fitness were measured by an oral glucose tolerance test, dual-energy $\mathrm{X}$-ray absorptiometry, magnetic resonance imaging, and a $\mathrm{VO}_{2}$ peak exercise test, respectively.

\section{Results}

The improvements in insulin AUC, body fat, visceral fat, and aerobic fitness in the exercise groups were significantly larger than the changes observed in the controls. Moreover, there were better improvements in insulin AUC, body fat, and visceral fat in the high-dose exercise group compared with the low-dose exercise group. However, these differences between 20 and $40 \mathrm{~min} /$ day did not reach statistical significance in all parameters measured. Similar fitness gains were observed in the two exercise groups. The adjusted mean changes for body mass index (BMI) $z$-scores and subcutaneous abdominal fat were significantly higher in the high-dose exercise group than in the low-dose exercise group $(-0.05 ; 95 \%$ confidence interval $[\mathrm{CI}],-0.10$ to $-0.01 ; p=0.02$, and $-8.62 ; 95 \% \mathrm{CI},-17.2$ to $-0.08 ; p=0.048$ ).

\section{Conclusions}

Supervised aerobic exercise for 20 or $40 \mathrm{~min} /$ day for 13 weeks was shown to improve insulin sensitivity, fitness, and adiposity in sedentary obese and overweight children, thus reducing the risk of developing T2DM.

\section{Comment}

Combined aerobic and resistance exercise is thought to be best for adults living with T2DM $(3,4)$. However, sufficient information is not readily available on the optimal prescription of exercise for youth at elevated risk of developing the disease. Given the increasing prevalence of childhood T2DM (5), understanding the dose-response relationship between aerobic exercise and T2DM risk factors in children is invaluable. Furthermore, this new research should enable health professionals to provide better exercise recommendations to children to reduce probability of developing T2DM. This study shows that with increasing exercise duration from 20 to $40 \mathrm{~min} /$ day, there is a decreasing trend in many of the risk factors for developing T2DM in youth aged 7-11 years. Future studies should be conducted to further examine the duration, type, and intensity of exercise that will result in maximal benefits to reducing T2DM risks in children.

\section{Effects of Tai Chi exercise on glucose control, neuro- pathy scores, balance, and quality of life in patients with type 2 diabetes and neuropathy}

Ahn S, Song $R$

College of Nursing, Chungnam National University, Daejeon, South Korea

J Altern Complement Med 2012; 18: 1172-78 


\section{Background and Rationale}

Diabetic peripheral neuropathy (DPN) is highly prevalent among patients with diabetes. It has debilitating effects on quality of life by impairing stability and increasing risk of falling. A small number of observational studies have shown that Tai Chi is beneficial for patients from a glucose control perspective, although its effect on DPN is unclear. The focus of this study is on Tai Chi's impact on DPN. Glucose control, peripheral sensory function, balance, and quality of life were measured to evaluate potential benefits.

\section{Methods}

A 12-week prospective study was conducted on 59 patients with diabetes type 2 and neuropathy, of which 39 patients completed the study. Average age of the subjects was 65 years. Data were analyzed based on 20 patients in the intervention group and 19 patients in the control group. Enzymatic assay, turbidimetric immunoassay, and Semmes-Weinstein monofilament examination and total neuropathy symptom scores were utilized to measure fasting blood glucose, $\mathrm{HbA} 1 \mathrm{c}$ levels, and peripheral sensory function, respectively. Balance was measured by the amount of time subjects could stand on one leg. The quality of life was measured by the Korean version of the 36-Item Short Form Health Survey. In this quasiexperimental design, subjects were not randomly assigned to groups. However, there were no significant differences in neuropathy scores, balance, glucose control, and quality of life between the groups at baseline.

\section{Results}

Posttests showed that glucose control (mean value changed from 137 to $125 \mathrm{mg} / \mathrm{dL}$ in the treatment group vs. 143 to $155 \mathrm{mg} / \mathrm{dL}$ for the controls; $t=2.23 ; p=0.036$ ), HbA1c (mean value changed from $7.63 \%$ to $7.20 \%$ in the treatment group vs. $8.02 \%$ to $8.32 \%$ for the controls; $t=3.11 ; p=0.004)$, balance (mean value changed from 22.37 to $30.02 \mathrm{sec}$ for single-leg stance in the treatment group vs. 15.71 to 14.27 in the controls), and total symptom scores were significantly better in the intervention group than in the control (mean change from 0.86 to 0.91 in the treatment group vs. 1.19 to 2.83 for the controls; $t=2.09 ; p=0.042$ ).

\section{Conclusion}

DPN is a common comorbidity of diabetes that leads to many complications, such as falls, injury, and reduced exercise adherence. Previous studies lasting longer than 12 weeks showed vascular improvements in diabetic patients with Tai Chi exercise. In this 12-week Tai Chi program, it was shown that this type of exercise modality helps improve glucose control, balance, and symptom scores of neuropathy in patients with T2DM. The authors note that Tai Chi shows great potential in terms of limiting the prognosis of diabetes.

\section{Comment}

DPN is a long-term complication frequently faced by the diabetic population. Since elderly patients are more likely to have had diabetes for a long time and therefore suffer from DPN, it is wise to study DPN treatment options that are suitable for this population. In this study, Tai Chi was shown to be very effective in improvement of neuropathic symptoms and in reduction of blood glucose levels. Regardless of the diabetes status, balance and falls are a common concern for the elderly, although the concern is more prominent in the diabetic population because is results in further postural instability. This study illustrates that Tai Chi is effective in improving balance in this patient group. One drawback of the study was the nonrandom assignment of subjects to the groups. Even though there was no significant difference between the groups at baseline, a clear trend was observed. Specifically, at baseline, subjects who would participate in Tai Chi had better scores in almost all study variables. Nevertheless, it should be concluded that Tai Chi is an effective exercise for treating complications associated with diabetes. It may be a simple and fun mode of exercise that is practical for almost everyone!

\section{Differences in the acute effects of aerobic and resistance exercise in subjects with type 2 diabetes: results from the RAED2 randomized trial}

Bacchi $E^{1}$, Negri $C^{1}$, Trombetta $M^{1}$, Zanolin $M E^{2}$, Lanza $M^{3}$, Bonora $E^{1}$, Moghetti $P^{1}$

${ }^{1}$ Section of Endocrinology, Diabetes and Metabolism, Department of Medicine, University and Azienda Ospedaliera Universitaria Integrata of Verona, Verona, Italy; ${ }^{2}$ Section of Epidemiology and Medical Statistics, Department of Public Health and Community Medicine, University of Verona, Verona, Italy; and ${ }^{3}$ Section of Motor Sciences, Department of Neurological, Neuropsychological, Morphological and Movement Sciences, University of Verona, Verona, Italy

\section{PLoS One 2012; 7:e49937}

\section{Background and Rationale}

It has been shown in previous studies that $\mathrm{HbA1c}$ levels are reduced with chronic aerobic or resistance exercise. This improvement in glycemic control is thought to be caused by increases in non-insulin-mediated glucose disposal during the exercise and by increased insulin sensitivity in resting muscle. Additive reduction of blood glucose by antidiabetic medications may increase the risk of hypoglycemia. Therefore, understanding the acute effects of exercise on blood glucose levels will allow appropriate modifications to diet and/or medication tailored to the exercise prescription, if needed. The objective of this study was to investigate the acute effects of aerobic and resistance exercise on blood glucose levels.

\section{Methods}

Twenty-five patients with T2DM participated in this randomized control trial over an $\sim 12$-week training period. Group 1 did aerobic exercise, while group 2 did resistance exercise. A continuous glucose monitoring system was inserted after $10.9+0.4$ weeks of training to study blood glucose levels during exercise and over the following 47 hours. 


\section{Results}

There were no significant differences in interstitial glucose AUC between the aerobic and the resistive exercise groups over the 48 hours after exercise. In the aerobic group, the glucose AUC during exercise was significantly lower than the corresponding period in the nonexercise day $(p=0.04)$. The trend held true for the nocturnal sleeping period that followed the exercise day and the corresponding period in the nonexercise day for the aerobic group $(p=0.02)$. Conversely, in the resistive exercise group, there were no significant differences in the glucose concentration AUC between exercise and nonexercise day and in the nocturnal sleeping period.

\section{Conclusions}

Both aerobic and resistance exercise reduce glucose exposure over the next 48 hours. However, there is a higher risk of late-onset nocturnal hypoglycemia with aerobic exercise compared with resistive exercise. This finding has important implications for medication prescription and dietary recommendations to the diabetic population. Also, caution should be taken when aerobic exercise is scheduled at times when late-onset hypoglycemia is likely to occur during sleep.

\section{Comment}

Prescription of exercise to diabetic patients is a common practice among health professionals. Physical activity guidelines have been established for the diabetic population; however, there is a need for understanding the effects of exercise in a nuanced way. This study sheds light on the acute effects of exercise on glycemia in the type 2 population. Acute aerobic exercise exhibits a higher likelihood of causing late-onset hypoglycemia than resistive exercise, similar to what has recently been reported for patients with T1DM (6). This finding is vital to exercise induced hypoglycemia prevention, especially during nocturnal periods. Unfortunately, there is no indication that the aerobic exercise group and the resistive exercise group performed an equivalent amount of exercise. Therefore, there is a potential for exercise volume to be a confounding variable. Further research should be done where comparisons of equivalent amounts of work (energy expenditure) are performed. Moreover, studies should investigate the intensity and duration of aerobic exercise that is least likely to cause hypoglycemia.

\section{Metabolomics approach for analyzing the effects of exercise in subjects with type 1 diabetes mellitus}

Brugnara $L^{1,2}$, Vinaixa $M^{2,3}$, Murillo $S^{1,2}$, Samino $S^{3,4}$, Rodriguez $M A^{2,3}$, Beltran $A^{\prime 3,4}$, Lerin $C^{1,2}$, Davison $G^{5}$, Correig $X^{2-4}$, Novials $A^{1,2}$

${ }^{1}$ Department of Endocrinology, Institut d'Investigacions Biomèdiques August Pi i Sunyer (IDIBAPS), Hospital Clinic de Barcelona, Barcelona, Spain; ${ }^{2}$ Spanish Biomedical Research Centre in Diabetes and Associated Metabolic Disorders (CIBERDEM), Barcelona, Spain; ${ }^{3}$ Metabolomics Platform, Universitat Rovira i Virgili, Tarragona, Spain; ${ }^{4}$ Institut d'Investigació Sanitària Pere Virgili (IISPV), Reus, Spain; and ${ }^{5}$ Sport and Exercise Sciences Research Institute, University of Ulster, Newtownabbey, Northern Ireland, United Kingdom

PLoS One 2012; 7:e40600

\section{Background and Rationale}

Exercise is commonly prescribed to T1DM patients; however, it may be causing metabolic disturbances. There is a lack of knowledge in the metabolic responses of T1DM population to exercise. The aim of this study was to investigate metabolic responses to acute exercise in T1DM patients and compare these responses with the responses in healthy controls. Understanding metabolic responses to acute exercise is valuable and will allow exercise prescription with higher accuracy and safety.

\section{Methods}

Ten men with T1DM and 11 healthy men who were active in their leisure time participated in this study. They were matched for age, BMI, body fat composition, and cardiorespiratory capacity. The pretest consisted of weight, height, BMI, total fat, and body fat composition measurements as well as filling out the International Physical Activity Questionnaire to show baseline activity level. The subjects were also required to do a maximum oxygen uptake test to establish a baseline fitness level. A week later, the subjects performed an acute bout of exercise at $80 \%$ of their VO2max for 30 minutes. All subjects fasted overnight for the preceding 12 hours of the test. Blood samples were taken from subjects before and after the exercise bout. Glucose-oxidase method and quimioluminiscent method were used to detect serum glucose and insulin levels, respectively. Serum proton nuclear magnetic resonance (H-NMR) and gas chromatography-mass spectrometry (GC-MS) were performed for the metabolite analysis.

\section{Results}

Before the exercise intervention, glucose, insulin, tricarboxylic acid (TCA) intermediates, and glycerol were higher and lysine was lower in the diabetic group compared with the control. After the exercise bout, glucose decreased and insulin increased significantly in the diabetic group, whereas there were no significant changes in the control. Gluconeogenic precursors elevated in both groups; however, the elevation was higher in the control. The same trend was observed with TCA cycle intermediates. Significant increase in lipolytic products was observed only in the control, with no significant changes in the diabetic group. Branched chain amino acids were significantly reduced in the control group only. Alphaketoisocaproic acid was significantly increased only in the control group. Lysine was significantly increased only in diabetic group.

\section{Conclusions}

Overall, the metabolic response to high-intensity acute exercise was similar in the T1DM group and the control. However, the T1DM group showed a weaker response than their control counterparts. Subjects in the T1DM group showed an elevation in serum lactate, whereas the control group showed an increase in lactate and pyruvate in response to exercise. This finding suggests that glycogenolysis and glycolysis are less activated after exercise in the T1DM group than in the control. This may be explained by the increased insulin in the T1DM group compared with the control. The reduced pyruvate in T1DM patients in 
response to exercise may be responsible for the reduced TCA cycle replenishment. Lipolysis is also lower in the T1DM group than in the control in response to exercise. These findings may suggest an impaired oxidative aerobic system in T1DM patients. Furthermore, data from this study suggest that protein catabolism is also attenuated in T1DM patients.

\section{Comment}

Although regular exercise is beneficial for most patients with T1DM (7), metabolic disturbances may be caused by acute exercise that may make glucose control challenging. In this study, although the patients with T1DM could all do the exercise task (intense cycling for 30 minutes), their fuel mobilization and utilization of energy differed somewhat compared with the age-matched controls. In general, it would appear from this study that at the onset of strenuous exercise, patients with T1DM have a sluggish breakdown of stored liver and muscle glycogen, a reduced energy flux through the TCA cycle, and reduced rates of adipose mobilization. It may be that the failure to lower circulating insulin levels at the onset of exercise promotes these disturbances in fuel mobilization. Nonetheless, the job still can get done!

\section{Short-term exercise training improves insulin sensitivity but does not inhibit inflammatory pathway in immune cells from insulin-resistant subjects}

Reyna $S M^{1-3}$, Tantiwong $P^{2,3}$, Cersosimo $E^{2,3}$, DeFronzo $R A^{2,3}$, Sriwijitkamol $A^{2}$, Musi $N^{2-4}$

${ }^{1}$ Medical Research Division, Regional Academic Health Center, Edinburg, TX; ${ }^{2}$ Diabetes Division, University of Texas Health Science Center at San Antonio, San Antonio, TX; ${ }^{3}$ Texas Diabetes Institute, San Antonio, TX; and ${ }^{4}$ Geriatric, Research, Education, and Clinical Center, Audie L. Murphy VA Hospital, San Antonio, TX

J Diabetes Res 2013; 2013: 107805

\section{Aim}

To determine if exercise improves insulin sensitivity in insulin-resistant subjects by downregulating proinflammatory signaling in immune cells.

\section{Methods}

The study included 17 lean, 8 obese nondiabetic, and 11 obese subjects with T2DM. Five T2DM subjects were diettreated, three T2DM subjects were newly diagnosed, and one T2DM participant was diagnosed 2 months before the study. Three months before the study, the subjects did not exercise or only exercised once during the week, had a stable body weight varying only by $1 \mathrm{~kg}$ for 3 months before the study, and underwent an insulin clamp procedure within 1-2 weeks after measuring subjects' baseline VO2peak. The subjects participated in a supervised exercise program within 1 week of the insulin clamp. The program consisted of a 40-minute cycle ergometer exercise for 15 consecutive days. The subjects' blood samples were collected before and after the pre- and post-exercise insulin clamps to isolate the peripheral blood mononuclear cells (PMNC). Toll-like receptors 4 and 2 (TLR4 and TLR2) activity were detected with primary and secondary antibodies using enhanced chemiluminescence reagents. Using Western blotting, researchers measured extracellular signal-regulated kinase (ERK) phosphorylation; C-Jun amino-terminal kinase (JNK) was measured as well. An enzyme-linked immunosorbent assay (ELISA) kit measured nuclear factor-kappa-light-chain-enhancer of activated $\mathrm{B}$ cells ( $\mathrm{NF} \kappa \mathrm{B}$ p65) binding, which plays a key role in regulating the immune response to infection.

\section{Results}

Subjects with T2DM had the highest fasting glucose levels, inflammatory, and endothelial markers. Obese and subjects with T2DM had a higher fasting insulin level, $\mathrm{HbA} 1 \mathrm{c}$, and non-esterified fatty acid (NEFA) concentration than the control lean group. Based on the lower total glucose disposal using the insulin clamp, diabetic and obese populations had more insulin resistance than the lean population. The diabetic and obese groups did not significantly decrease BMI or weight with the increase in exercise. Also, no change occurred in $\mathrm{HbA1c}$, NEFA, glucose, and plasma insulin levels. After exercise, insulin sensitivity increased by $11 \%$ in lean, $15 \%$ in obese, and $32 \%$ in T2DM groups $(p<0.05$ in all groups). TLR4 protein (activates immune system) levels in the PMNC subjects with diabetes were 4.2-fold higher compared with the lean subjects $(p<0.05)$. TLR4 was 2.7 -fold $(p=0.07)$ higher in the obese than in the lean group. Neither exercise training nor insulin therapy changed LR2 levels in the PMNC group. Diabetic subjects also had higher baseline ERK phosphorylation, but hyperinsulinemia during the insulin clamp did not significantly affect ERK phosphorylation. JNK phosphorylation was not affected by insulin infusion or exercise. Neither insulin nor exercise affected $\mathrm{NF} \kappa \mathrm{B}$ p65 DNA binding in the lean, obese, and T2DM subjects.

\section{Conclusions}

PMNC cells were isolated because they fight infection and adapt to intruders. These cells allowed the research to conclude that diabetic patients have increased proinflammatory signaling, shown by increased TLR4 and ERK phosphorylation. TLR4, TLR2 are important in the activation of the innate immune system, while ERK phosphorylation refers to a signaling pathway composed of proteins and enzymes involved in cellular processes, mainly cell survival and apoptosis. ERK deletion has been shown to improve insulin sensitivity in skeletal muscle and reduce liver fat content (8). While exercise improved insulin sensitivity in the lean, obese, and T2DM groups, it did not help with the proinflammatory state, as measured by TLR content and ERK phosphorylation. Since proinflammatory monocytes adhere more strongly to activated endothelial cells and are precursors to the CD16 + macrophages distributed in atherosclerotic lesions, changes in TLR4 protein content and ERK signaling may have affected the results. Contrary to studies in mice that suggest that JNK plays an important role in the pathogenesis of obesity and insulin resistance (9), the JNK phosphorylation was not affected by insulin infusion nor exercise. 


\section{Comment}

This research shows that exercise is beneficial for insulin sensitivity but not for downregulating proinflammatory signaling in immune cells. It also suggests that insulin sensitivity and inflammation are not tightly coupled. This study is a stepping stone for further research in linking the immune response with insulin resistance and exercise not only because of the interesting results, but also because it references at least two studies that either supported or conflicted with each finding.

\section{Cardiovascular effects of intensive lifestyle intervention in type 2 diabetes}

Look AHEAD Research Group, Wing $R R^{1}$, Bolin $P^{2}$, Brancati $F L^{3}$, Bray $G A^{4}$, Clark $J M^{5}$, Coday $M^{6}$, Crow $R S^{7}$, Curtis $J M^{8}$, Egan $\mathrm{CM}^{9}$, Espeland $M A^{10}$, Evans $M^{11}$, Foreyt $J P^{12}$, Ghazarian $S^{13}$, Gregg $E W^{14}$, Harrison $B^{15}$, Hazuda $\mathrm{HP}^{16}$, Hill $\mathrm{JO}^{17}$, Horton ES ${ }^{18}$, Hubbard VS ${ }^{19}$, Jakicic $J M^{20}$, Jeffery $R W^{2}$, Johnson $K^{22}$, Kahn $S E^{23}$, Kitabchi $A E^{24}$, Knowler $W C^{25}$, Lewis $C E^{26}$,

Maschak-Carey $B J^{27}$, Montez $\mathrm{MG}^{28}$, Murillo $A^{29}$, Nathan $D^{30}$, Patricio $J^{31}$, Peters $A^{32}$, Pi-Sunyer $X^{33}$, Pownall $H^{34}$, Reboussin $D^{35}$, Regensteiner $J G^{36}$, Rickman $A D^{37}$, Ryan $D H^{38}$, Safford $M^{39}$, Wadden $T A^{40}$, Wagenknecht $L E^{41}$, West DS ${ }^{42}$, Williamson $D F^{43}$, Yanouski $\mathrm{SZ}^{44}$

${ }^{1}$ Weight Control and Diabetes Research Center, Warren Alpert Medical School of Brown University and Miriam Hospital, Providence, RI; ${ }^{2}$ National Institute of Diabetes and Digestive and Kidney Diseases, National Institutes of Health, Bethesda, MD; ${ }^{3} J o h n s$ Hopkins School of Medicine, Baltimore, $M D ;{ }^{4}$ Pennington Biomedical Research Center, Baton Rouge, LA; ${ }^{5}$ Johns Hopkins School of Medicine, Baltimore, MD; ${ }^{6}$ Department of Preventive Medicine, University of Tennessee Health Sciences Center, Memphis, TN; ${ }^{7}$ Division of Epidemiology and Community Health, University of Minnesota, Minneapolis, MN; ${ }^{8}$ NIH/NIDDK Southwest American Indian Center; ${ }^{9}$ Weight Control and Diabetes Research Center, Warren Alpert Medical School of Brown University and Miriam Hospital, Providence, $R I_{;}{ }^{10}$ Department of Biostatistical Sciences, Wake Forest University School of Medicine, Winston-Salem, NC; ${ }^{11}$ NIH/NIDDK, Bethesda, MD; ${ }^{12}$ Department of Medicine, Baylor College of Medicine, Houston, TX; ${ }^{13}$ Roybal Comprehensive Health Center, Los Angeles, $C A ;{ }^{14}$ Centers for Disease Control and Prevention, Atlanta, GA; ${ }^{15} \mathrm{NIH} /$ NIDDK, Bethesda, MD; ${ }^{16}$ Department of Clinical Epidemiology, University of Texas Health Science Center at San Antonio, San Antonio, TX; ${ }^{17}$ Center for Human Nutrition, University of Colorado Health Sciences Center, Aurora, CO; ${ }^{18}$ Joslin Diabetes Center, Boston, MA; ${ }^{19}$ NIH/NIDDK, Bethesda, MD; ${ }^{20}$ Department of Health and Physical Activity, University of Pittsburgh, Pittsburgh, PA; ${ }^{21}$ Division of Epidemiology and Community Health, University of Minnesota, Minneapolis, $M N_{;}{ }^{22}$ Department of Preventive Medicine, University of Tennessee Health Sciences Center, Memphis, TN; ${ }^{23}$ Department of Medicine, University of Washington, Seattle, WA; ${ }^{24}$ Department of Preventive Medicine, University of Tennessee Health Sciences Center, Memphis, TN; ${ }^{25}$ NIH/NIDDK Southwest American Indian Center, Phoenix, AZ; ${ }^{26}$ Division of Preventive Medicine, University of Alabama at Birmingham, Birmingham, AL; ${ }^{27}$ Weight and Eating Disorder Program, University of Pennsylvania, Philadelphia, PA;
${ }^{28}$ Department of Clinical Epidemiology, University of Texas Health Science Center at San Antonio, San Antonio, TX; ${ }^{29}$ Department of Medicine, University of Washington, Seattle, WA; ${ }^{30}$ Diabetes Unit, Massachusetts General Hospital, Boston, MA; ${ }^{31}$ Department of Medicine, St. Luke's-Roosevelt Hospital, New York, NY; ${ }^{32}$ Roybal Comprehensive Health Center, Los Angeles, $C A ;{ }^{33}$ Department of Medicine, St. Luke's-Roosevelt Hospital, New York, NY; ${ }^{34}$ Department of Medicine, Baylor College of Medicine, Houston, TX; ${ }^{35}$ Department of Biostatistical Sciences, Wake Forest University School of Medicine, Winston-Salem, NC; ${ }^{36}$ Center for Women's Health Research, University of Colorado Health Sciences Center, Aurora, CO; ${ }^{37}$ Department of Health and Physical Activity, University of Pittsburgh, Pittsburgh, PA; ${ }^{38}$ Pennington Biomedical Research Center, Baton Rouge, LA; ${ }^{39}$ Division of Preventive Medicine, University of Alabama at Birmingham, Birmingham, AL; ${ }^{40}$ Weight and Eating Disorder Program, University of Pennsylvania, Philadelphia, PA; ${ }^{41}$ Division of Public Health Sciences, Wake Forest University School of Medicine, Winston-Salem, NC; ${ }^{42}$ Department of Health Behavior and Health Education, College of Public Health, University of Arkansas for Medical Sciences, Little Rock, AR; ${ }^{43}$ Centers for Disease Control and Prevention, Atlanta, GA; ${ }^{44}$ NIH/NIDDK, Bethesda, MD

N Engl J Med 2013; 369: 145-54

\section{Background and Rationale}

To determine whether a long-term intensive lifestyle intervention for weight loss decreases cardiovascular morbidity and mortality for overweight or obese patients with T2DM.

\section{Methods}

The study followed 5,145 overweight or obese T2DM patients for up to 13.5 years. Each patient was randomly assigned to an intervention (targeting a $7 \%$ loss in body weight) or control group. The intervention group emphasized weight loss through decreased caloric intake and increased physical activity, while the control group emphasized diabetes support and education.

\section{Results}

The intervention group had greater weight loss than the control group $(8.6 \%$ vs. $0.7 \%$ at 1 year, $6.0 \%$ vs. $3.5 \%$ at study end). Additionally, the intervention group reduced glycated hemoglobin and initially improved fitness and all cardiovascular risk factors, besides low-density-lipoprotein cholesterol levels, more so than the control group. The primary outcome (composite of death from cardiovascular causes, nonfatal myocardial infarction, nonfatal stroke, or hospitalization for angina) occurred in 403 intervention patients and in 418 control patients. The trial was stopped early (after a follow-up of $\sim 9$ years) when interim analyses suggested that the intervention was having a neutral effect on the primary outcome.

\section{Conclusions}

The long-term intensive lifestyle intervention focusing on weight loss failed to reduce the rate of cardiovascular events in overweight or obese adults with T2DM. However, the intervention group had greater weight loss, reductions in glycated hemoglobin, and greater initial improvements in fitness and cardiovascular risk factors than the control group. 


\section{Comment}

These findings appear to suggest that lifestyle interventions do not effectively reduce the rate of cardiovascular events in patients with T2DM. This is surprising since a plethora of studies support the recommendation for overweight or obese patients to improve health status by losing weight with diet and exercise. A short-term 16-week study indicated that a very-low-calorie diet improved the cardiovascular risk profile (metabolic profile, heart function, and triglyceride stores in nonadipose tissues) in adults with T2DM (10). This also counters observational studies that have been reported that greater fitness is associated with reduced risk of developing T2DM $(11,12)$. The long followup period implemented in this study may have been the key factor in achieving such a different result. This study employed an interesting angle by educating the support group. Overall, the cardiovascular events were relatively low in both groups (all patients were aggressively treated with cardioprotective drugs) and thus detecting differences between the two intervention groups may have been difficult in the period studied.

\section{Correlations of non-exercise activity thermogenesis to metabolic parameters in Japanese patients with type 2 diabetes}

Hamasaki $H^{1,2}$, Yanai $H^{1}$, Mishima $S^{1}$, Mineyama $T^{1}$, Yamamoto-Honda $R^{3,4}$, Kakei $M^{2,5}$, Ezaki $\mathrm{O}^{3,6}$, Noda $M^{3,4}$

${ }^{1}$ Department of Internal Medicine, National Center for Global Health and Medicine Kohnodai Hospital, Chiba, Japan; ${ }^{2}$ General Internal Medicine, Community Healthcare Studies, Jichi Medical University Graduate School, Tochigi, Japan; ${ }^{3}$ Department of Diabetes and Metabolic Medicine, Center Hospital, National Center for Global Health and Medicine, Tokyo, Japan; ${ }^{4}$ Department of Diabetes Research, Diabetes Research Center, National, Center for Global Health and Medicine, Tokyo, Japan; ${ }^{5}$ First Department of Comprehensive Medicine, Saitama Medical Center, Jichi Medical University School of Medicine, Saitama, Japan; and ${ }^{6}$ Department of Human Health and Design, Faculty of Human Life and Environmental Sciences, Showa Women's University, Tokyo, Japan

Diabetol Metab Syndr 2013; 5: 26

\section{Background and Rationale}

To determine how non-exercise activity thermogenesis (NEAT) is associated with cardiovascular risk factors in patients with T2DM. The NEAT score is the energy expenditure due to physical activities other than planned exercise (i.e., sports, planned exercise) in daily life. Examples include commuting to work, attending school, singing, dancing, washing clothes, and cleaning.

\section{Methods}

The researchers questioned 45 subjects about their physical activity patterns using an original NEAT questionnaire that evaluated physical activity habits. The subjects consisted of 22 women and 23 men with T2DM, aged between 20 and 90 years, who did not take any hypoglycemic, antihypertensive, or cholesterol-lowering agents. Subjects who engaged in active sports-like exercise and resistance training were excluded from the study. As outlined by Ainsworth and colleagues' guidelines (13), each questionnaire item was given a score of 1-3 points in order of levels of habitual physical activity, and then the scores were added to determine the overall NEAT score. This score was then compared with the subjects' body weight, waist circumference, blood pressure, glucose and lipid metabolism, and arterial stiffness using Pearson's correlation coefficients.

\section{Results}

The NEAT score was negatively correlated with serum insulin levels (all subjects) and was negatively correlated with waist circumference and positively correlated with highdensity lipoprotein cholesterol levels in women. The NEAT score was negatively associated with serum insulin levels and systolic and diastolic blood pressure in patients with abdominal obesity.

\section{Conclusion}

A high NEAT score is associated with benefiting insulin sensitivity, waist circumference, high-density lipoprotein cholesterol, blood pressure, and atherosclerosis in patients with T2DM. Obese patients with both T2DM and hypertension had a significantly lower likelihood of adopting physical activity to control their weight compared with patients with neither condition ( $6 \%$ vs. $12 \%, p<0.01)(14)$. This implies that NEAT is more crucial for controlling body weight in obese patients with T2DM in comparison to those without T2DM.

\section{Comment}

This is a cross-sectional study limiting inferences of causality and its direction. The small sample size limited the study as well. For instance, the significant correlation between NEAT and serum insulin may have been highly influenced by three subjects with very high insulin and low NEAT. The primary drawback of this study was the subjective questionnaire, rather than using more objective measures of energy expenditure. Moreover, selfreports do not account for other potentially contributing factors, such as genetics or aerobic capacity, underlying both the increased NEAT scores and health variables measured. Particularly interesting to us was why only the female patients' NEAT score was negatively associated with waist circumference and positively associated with high-density lipoprotein cholesterol levels. Is it truly a sex-related phenomenon?

\section{Determinants of the changes in glycemic control with exercise training in type 2 diabetes: a randomized trial}

Johannsen NM, Sparks LM, Zhang Z, Earnest CP, Smith SR, Church TS, Ravussin E

Pennington Biomedical Research Center and Louisiana State University, Baton Rouge, LA

PLoS One 2013; 8: e62973

\section{Background and Rationale}

To examine the effects of chronic exercise training on total serum adiponectin, free fatty acid (FFA) concentrations, and skeletal muscle peroxisome proliferator-activated receptor- $\gamma$ 
coactivator-1 $\alpha$ (PGC-1 $\alpha$ ) protein content and their relationship to changes in glycemic control (HbA1c) in T2DM.

\section{Methods}

A subcohort of 35 patients (mean age $57.0 \pm 7.7$ years, $48 \%$ men, $74 \%$ Caucasian) from the Health Benefits of Aerobic and Resistance Training in Type 2 Diabetes study was analyzed. Participants were randomized to 9 months of aerobic training, resistance training, combination of both, or a nonexercise group. Muscle biopsies and serology were collected pre- and postintervention.

\section{Results}

Changes in $\mathrm{HbA1}$ c were inversely associated with changes in serum adiponectin levels $(r=-0.45, p=0.007)$. Participants diagnosed with T2DM for a longer duration had the largest increase in PGC- $1 \alpha \quad(r=0.44, p=0.008)$. Statistical modeling examining changes in $\mathrm{HbA1}$ c suggested that male sex $(p=0.05)$, non-Caucasian ethnicity $(p=0.02)$, duration of T2DM $(r=0.40$; $p<0.002)$, and changes in FFA $(r=0.36 ; p<0.004)$, adiponectin $(r=-0.26 ; p<0.03)$, and PGC- $1 \alpha(r=-0.28 ; p=0.02)$ levels explained $65 \%$ of the variability in the changes in $\mathrm{HbA} 1 \mathrm{c}$.

\section{Conclusion}

Changes in $\mathrm{HbA} 1 \mathrm{c}$ after 9 months of exercise were independently associated with the duration of T2DM and changes in serum FFA and negatively associated with changes in serum adiponectin and skeletal muscle PGC- $1 \alpha$.

\section{Comment}

T2DM is as much a disease of disordered lipid metabolism as a disease of abnormal glucose metabolism. Individuals with T2DM have an elevation in serum FFA and a reduction in plasma adiponectin and PGC- $1 \alpha$ expression in skeletal muscle. PGC- $1 \alpha$ is a key regulator of mitochondrial biogenesis and oxidative metabolism, which is critical in helping maintain muscle insulin sensitivity and efficient metabolism. Decreases in $\mathrm{HbA1c}$ after 9 months of exercise were associated with shorter duration of diabetes, lowering of serum FFA concentrations, increasing serum adiponectin concentrations, and increasing skeletal muscle PGC- $1 \alpha$ protein expression. A program of combined aerobic and resistance training has the greatest effect on HbA1c. Resistance training alone may potentiate a greater change in PGC- $1 \alpha$ and, therefore, HbA1c. Based on this study, individuals who start an exercise program soon after diagnosis of diabetes may see a larger effect on $\mathrm{HbA1c}$ levels. A prescription for exercise training programs for T2DM should be aimed at improving plasma substrate availability, endocrine function, and skeletal muscle factors shown to improve glycemic outcomes.

\section{Author Disclosure Statement}

No competing financial interests exist.

\section{References}

1. Yardley JE, Stapleton JM, Sigal RJ, Kenny GP. Do heat events pose a greater health risk for individuals with type 2 diabetes? Diabetes Technol Ther 2013; 15: 520-29.

2. Fang ZY, Sharman J, Prins JB, Marwick TH. Determinants of exercise capacity in patients with type 2 diabetes. Diabetes Care 2005; 28: 1643-48.

3. Grelier SF, Serresse OF, Boudreau-Lariviere CF, Zory R. Effects of a three-month combined training program on the cardiopulmonary and muscle strength capacities of type 2 diabetic subjects. J Sports Med Phys Fitness 2013; 53: $56-64$.

4. O'Hagan C, De Vito G, Boreham CA. Exercise prescription in the treatment of type 2 diabetes mellitus: Current practices, existing guidelines and future directions. Sports Med 2013; 43: 39-49.

5. Copeland KC, Silverstein J, Moore KR, et al. Management of newly diagnosed type 2 diabetes mellitus (T2DM) in children and adolescents. Pediatrics 2013; 131: 364.

6. Yardley JE, Kenny GP, Perkins BA, et al. Resistance versus aerobic exercise: acute effects on glycemia in type 1 diabetes. Diabetes Care 2013; 36: 537-42.

7. Chimen M, Kennedy A, Nirantharakumar K, et al. What are the health benefits of physical activity in type 1 diabetes mellitus? A literature review. Diabetologia 2012; 55: 542-51.

8. Jager J, Corcelle V, Grémeaux T, et al. Deficiency in the extracellular signal-regulated kinase 1 (ERK1) protects leptindeficient mice from insulin resistance without affecting obesity. Diabetologia 2011; 54: 180-89.

9. Hirosumi J, Tuncman G, Chang L, et al. A central role for JNK in obesity and insulin resistance. Nature 2002; 420: 333-36.

10. Snel M, Jonker JT, Hammer S, et al. Long-term beneficial effect of a 16-week very low calorie diet on pericardial fat in obese type 2 diabetes mellitus patients. Obesity (Silver Spring) 2012; 20: 1572-76.

11. Sui X, Hooker SP, Lee IM, et al. A prospective study of cardiorespiratory fitness and risk of type 2 diabetes in women. Diabetes Care 2008; 31: 550-55.

12. Tudor-Locke C, Bell RC, Myers AM, et al. Controlled outcome evaluation of the First Step Program: A daily physical activity intervention for individuals with type II diabetes. Int J Obes Relat Metab Disord 2004; 28: 113-19.

13. Ainsworth BE, Haskell WL, Herrmann SD, et al. Compendium of physical activities: A second update of codes and MET values. Med Sci Sports Exerc 2011; 5: 1575-81.

14. Zhao G, Ford ES, Li C, Mokdad AH. Weight control behaviors in overweight/obese U.S. adults with diagnosed hypertension and diabetes. Cardiovasc Diabetol 2009; 5: 13. 